submitted for publication to The Journal of Physical Chemistry B

\title{
Transferable Potentials for Phase Equilibria. 4. United-Atom Description of Linear and Branched Alkenes and Alkylbenzenes
}

\author{
Collin D. Wick, ${ }^{1}$ Marcus G. Martin, ${ }^{1,2}$ and J. Ilja Siepmann ${ }^{*}, 1$ \\ ${ }^{1}$ Departments of Chemistry and of Chemical Engineering and Materials Science, \\ University of Minnesota, 207 Pleasant St. SE, Minneapolis, MN 55455-0431 \\ and \\ ${ }^{2}$ Computational Biology and Materials Technology, \\ Sandia National Laboratory, Albuquerque, NM 87185-1111
}

* Corresponding author: siepmann@chem.umn.edu 


\begin{abstract}
The Transferable Potentials for Phase Equilibria-United Atom (TraPPE-UA) force field for hydrocarbons is extended to alkenes and alkylbenzenes by introducing the following pseudo-atoms: $\mathrm{CH}_{2}\left(\mathrm{sp}^{2}\right), \mathrm{CH}\left(\mathrm{sp}^{2}\right), \mathrm{C}\left(\mathrm{sp}^{2}\right), \mathrm{CH}($ aro $), \mathrm{R}-\mathrm{C}$ (aro) for the link to aliphatic side chains, and $\mathrm{C}($ aro $)$ for the link of two benzene rings. In this united-atom force field. the nonbonded interactions of the hydrocarbon pseudo-atoms are solely goremed by LemnardJones 12-6 potentials, and the Lennard-Jones well depth and size parameters for the new pseudo-atoms were determined by fitting to the single-component vapor-liquid phase equilibria of a few selected model compounds. Configurational-bias Monte Carlo simulations in the NVT version of the Gibbs ensemble were carried out to calculate the single-component vapor-liquid coexistence curves for ethene, propene, 1-butene, trans- and cis-2-butene. 2methylpropene, 1.5-hexadiene, 1-octene, benzene, toluene, ethylbenzene, propylbenzene: isopropylbenzene, $o-m$-, and p-xylene, and naphthalene. The phase diagrams for the binary mixtures of (supercritical) ethene/ $n$-heptane and benzene/n-pentane were determined from simulations in the $N p T$ Gibbs ensemble. Although the TraPPE-UA force field is rather simple and makes use of relatively few different pseudo-atoms, its performance. as judged by comparisons to other popular force fields and available experimental data. is very satisfactory.
\end{abstract}




\section{DISCLAIMER}

This report was prepared as an account of work sponsored by an agency of the United States Government. Neither the United States Government nor any agency thereof, nor any of their employees, make any warranty, express or implied, or assumes any legal liability or responsibility for the accuracy, completeness, or usefulness of any information, apparatus, product, or process disclosed, or represents that its use would not infringe privately owned rights. Reference herein to any specific commercial product, process, or service by trade name, trademark, manufacturer, or otherwise does not necessarily constitute or imply its endorsement, recommendation, or favoring by the United States Government or any agency thereof. The views and opinions of authors expressed herein do not necessarily state or reflect those of the United States Government or any agency thereof. 


\section{DISCLAIMER}

Portions of this document may be illegible in electronic image products. Images are produced from the best available original document. 


\section{Introduction}

Unsaturated hydrocarbons are ubiquitous as reactants. intermediates, or products in many processes in the (perro) chemical industries, and the rigidity and distinct conformation of unsaturated carbon-carbon bonds is exploited by many biological systems. However, despite the importance of unsaturated hydrocarbons little attention has been devoted to the development of appropriate molecular-mechanics-type force fields for these sistems that are transferable over a wide range of temperature, pressure, and composition.

The popular OPLS mited-atom force field developed by Jorgensen et al. ${ }^{1}$ contains the Lennard-Jones (LJ) parameters (and also the torsional parameters) for some of the pseudo-atoms required for modeling of unsaturated hydrocarbons. In particular, these OPLS-UA force field parameters were fitted to liquid densities, heats of vaporization, and liquid structures of 1-butene, cis-2-butene, 2-methylpropene, and benzene (at $T=298.15 \mathrm{~K}$ and $p=101.3 \mathrm{kPa}$ ) determined from isobaric-isothermal ensemble Monte Carlo simulations. Using a similar procedure, Jorgensen et al. ${ }^{2.3}$ also determined force field parameters (LJ well depths and LJ diameters plus partial charges) for an all-atom description of the unsaturated hydrocarbons. However, over the past few rears it has become clear that simulations over a wider range of physical conditions (temperature and pressure) are required to yield force fields for a given system that strike a good balance between enthalpic and entropic contributions to the free energy and that are thus transferable to different physical conditions. ${ }^{4,5}$

Recently, Spyriouni et al. ${ }^{6}$ proposed a united-atom model for medium-sized $\alpha$-olefines (denoted here as the SET force field) that yields very good predictions for many thermophysical properties (critical constants, vapor pressures, heats of vaporization, and second virial coefficients) over the entire fluid phase regime. To further test their $\alpha$-olefine force field, Spyriouni et al. ${ }^{6}$ carried out calculations for binary mixtures of 1-butene (or 1-hexene) 
with $n$-hexatriacontane which was modeled using the $n$-alkane force field of Dodd and Thereluron: 'In addition, in an earlier publications results for binary mixtures of ethene with heavy saturated alkanes were reported. Although their results for the pure olefines and their mixtures with $n$-alkanes are very satisfactory, we would like to emphasize here that the SET force field parameters used were not transferable from one type of molecule to another. In particular, the $\mathrm{CH}_{2}\left(\mathrm{sp}^{2}\right)$ parameters are different for this pseudo-atom in ethene and in the $\alpha$-olefines. and the saturated methylene and methyl group parameters differ between ethane, longer $n$-alkanes, and $\alpha$-olefines (see Table 1 ). This implies that one needs to determine all different types of pseudo-atoms separately for every class of molecules. and therefore could not use a group-contribution-like concept to build new molecules.

A few rears ago, this group has started an effort to develop the transferable potentials for phase equilibria (TraPPE) force fields. ${ }^{9-11}$ Saturated alkanes were the main focus in the first three papers in this series, and a computational efficient united-atom force field (TraPPE- $-A$ ) was developed for linear and branched alkanes. ${ }^{9,10}$ In addition, an explixithrdrogen version (TraPPE-EH) for the linear alkanes was presented that is computationally more expensive, but yields slightly better predictions for phase equilibrium properties. ${ }^{11}$

The purpose of the TraPPE force fields is not only to be able to reproduce thermophysical properties over a wide range of physical conditions, but also to keep the models as transferable as possible by minimizing the number of different (pseudo-)atoms needed for any particular molecule and by using the same parameters for a given (pseudo-)atom in all types of molecules. In particular, for the united-atom version this requires further explanation. Our philosophy in defining a given pseudo-atom is that it mimicks the interactions of its core electrons plus a share of the valence electrons that make its bonds to the neighboring atoms. Actually, the contribution of the valence electrons far outweighs the contribution of the core electrons to the molecular polarizabilty. ${ }^{12,13}$ For example, the 
pseudo-atom for a methyl group. that is comnected to another carbon atom. account: for the three $\mathrm{C}-\mathrm{H}$ bonds and a share of one $\mathrm{C}-\mathrm{C}$ bond (plus its core electrons). Thus the same pseudo-atom is used for the methyl group in ethane. propane, or propene. However. the pseudo-atom for a methyl group connected by a single bond to an oxygen atom (sav, in methanol) is different because it mimicks the interactions of three $\mathrm{C}-\mathrm{H}$ bonds and a share of one $\mathrm{C}-\mathrm{O}$ bond. Clearly differences in electronegativity between $\mathrm{C}$ and $\mathrm{O}$ atoms lead to intramolecular charge transfer and require the use of partial charges and. marbe, also of different LJ parameters. The next manuscript in this series will focus on oxygen functionalities. ${ }^{14}$

To model linear and branched alkenes and alkylbenzenes (and aromatic hydrocarbons consisting of multiple benzene rings) we need to introduce three new pseudo-atoms with $\mathrm{sp}^{2}$ geometry $\left[\mathrm{CH}_{2}\left(\mathrm{sp}^{2}\right), \mathrm{CH}\left(\mathrm{sp}^{2}\right)\right.$, and $\left.\mathrm{C}\left(\mathrm{sp}^{2}\right)\right]$ and three new aromatic pseudo-atoms $\mathrm{CH}($ aro $), \mathrm{R}-\mathrm{C}($ aro $)$ for the link to aliphatic side chains, and $\mathrm{C}($ aro $)$ for the link of benzene rings]. The remainder of this manuscript is organized as follows. The details of the TraPPE-UA force field for alkenes and alkylbenzenes are described in the next section. Thereafter, we briefly outline the simulation methods. Results for vapor-liquid equilibria of pure systems and binary mixtures are discussed in section 4 .

\section{Models}

The TraPPE-UA force field utilizes pseudo-atoms located at the sites of heary atoms (in this case, only carbon atoms) whose nonbonded interactions are described by pairwiseadditive LJ $12-6$ potentials

$$
u_{\mathrm{LJ}}\left(r_{i j}\right)=4 \epsilon_{i j}\left[\left(\frac{\sigma_{i j}}{r_{i j}}\right)^{12}-\left(\frac{\sigma_{i j}}{r_{i j}}\right)^{6}\right]
$$

where $r_{i j}, \epsilon_{i j}$, and $\sigma_{i j}$ are the bead-bead separation, LJ well depth, and LJ diameter, respectively, for the beads $i$ and $j$. As is customary, these LJ potentials are used only 
for the interactions of pscudo-atoms belonging to different molecules or belonging to the same molecule, but whose interactions are not accounted for by any of the intramolecular: bonded potentials: fixed bond lengths for neighboring pseudo-atoms, harmonic bond bending potentials for pseudo-atoms separated by two bonds, and dihedral potentials for pseudo-atoms separated by three flexible bonds. Since the benzene rings are treated here as rigid units, the dihedral potential for a pseudo-atom that is in $\beta$ position (separated by two bonds) to a benzene ring accounts for all the 1-4, 1-5 and 1-6 interactions with the pseudo-atoms of the benzene ring. Furthermore, napthalene is treated as a fully rigid molecule and therefore all intramolecular LJ interactions are excluded.

The LJ parameters for methyl, methylene, and methine groups with $\mathrm{sp}^{3}$ can be taken directly taken from our work on linear and branched alkanes, ${ }^{9,10}$ since they are always bonded to another carbon atom for all molecules studied here. The new LJ parameters for the vinylic groups were determined from calculations of the vapor-liquid equilibria of the following compounds: ethene for $\mathrm{CH}_{2}\left(\mathrm{sp}^{2}\right)$, cis-2-butene for $\mathrm{CH}\left(\mathrm{sp}^{2}\right), 2$-methylpropene for $\mathrm{C}\left(\mathrm{sp}^{2}\right)$, benzene for $\mathrm{CH}($ aro $)$, toluene for $\mathrm{R}-\mathrm{C}(\operatorname{aro})$, and napthalene for $\mathrm{C}(\operatorname{aro})$. The set of TraPPE-UA LJ parameters used here and the corresponding parameters for the OPLS-UA model developed by Jorgensen et al. ${ }^{1}$ and the different models by Theodorou and co-workers ${ }^{6-8}$ are listed in Table 1 . The $\mathrm{LJ}$ parameters for the interactions of unlike pseudo-atoms are calculated using the standard Lorentz-Berthelot combining rules ${ }^{15,16}$

$$
\sigma_{i j}=\frac{1}{2}\left(\sigma_{i i}+\sigma_{j j}\right) \quad \epsilon_{i j}=\sqrt{\epsilon_{i i} \epsilon_{j j}}
$$

A spherical potential truncation for pairs of pseudo-atoms separated by more than $14 \AA$ was enforced, and analytic tail corrections (for energies, pressures, and chemical potentials) were employed. ${ }^{17,18}$ For the saturated and unsaturated hydrocarbons, all pseudo-atoms are connected to pseudo-atoms with the heavy atoms of the same electronegativity, thus partial charges are not needed here for the TraPPE-UA force field. Similarly, partial charges are 
not used in the OPLS-UA representation, ${ }^{1}$ whereas the OPLS-AA model for benzene ${ }^{2}$ makes use of partial charges at the carbon and lyydrogen sites. In this case. the Ewald summation technique is employed to calculate the long-range Coulombic interactions with the Ewald parameters set to $\kappa \times L=5$ and $K_{\max }=5$.

As mentioned above, a set of bonded interactions governs bond stretching, bond angle bending, and torsional motions. In the TraPPE-UA force field all bond lengths are fixed and aromatic rings are treated as rigid units. The bond lengths for the three different types of carbon-carbon bonds (single bond, double bond, aromatic ring bond) are listed in Table 2.

A harmonic potential is used to control bond angle bending

$$
u_{\text {bend }}=\frac{k_{\theta}}{2}\left(\theta-\theta_{0}\right)^{2}
$$

where $\theta, \theta_{0}$, and $k_{\theta}$ are the measured bending angle, the equilibrium bending angle, and the force constant, respectively. Table 3 contains a list of the $\theta_{0}$ and $k_{\theta}$ parameters ${ }^{19,20}$ used here (see also ref. 10 for additional TraPPE-UA bending parameters).

The torsional potentials used to restrict the dihedral rotations around bonds connecting two methylene pseudo-atoms and around bonds connecting a methylene pseudo-atom with a $\mathrm{CH}\left(\mathrm{sp}^{2}\right)$ were taken from the OPLS-UA force field ${ }^{1}$

$$
u_{\mathrm{tors}}=c_{0} c_{1}[1+\cos (\phi)]+c_{2}[1-\cos (2 \phi)]+c_{3}[1+\cos (3 \phi)]
$$

with the Fourier coefficients listed in Table 4 (additional torsional potentials for TraPPEUA branched alkanes can be found in ref. 10).

Although trans- and cis-2-butene are treated as rigid molecules in the OPLS-UA force field. a harmonic torsional potential is used for the TraPPE-UA force field

$$
u_{\text {tors }}=\frac{d_{0}}{2}\left(\phi-\phi_{0}\right)^{2}
$$


In this case. the $d_{0}$ and $\phi_{0}$ parameters (see Table 4 ) were obtained by fitting to the energic: of fully relaxed structures calculated with the MIMx representation of PC-.Iodel. ${ }^{21} \mathrm{~A}$ simple cosine function is used for dihedral rotations around bonds connecting an $\mathrm{sp}^{3}$ carbon with an aromatic carbon

$$
u_{\text {tors }}=e_{0}\left[1-\cos \left(2 \dot{0}+e_{1} !\right.\right.
$$

with parameters derived using PC-Model (see Table 4).

\section{Simulation Details}

A combination ${ }^{22}$ of the Gibbs ensemble Monte Carlo (GEMC) technique $23-25$ and the configurational-bias Monte Carlo (CBMC) method ${ }^{10.26-29}$ was used to determine the vapor-liquid coexistence properties for single-component systems and binary mixtures. To facilitate the particle swap moves that are required to sample the partitioning of molecules between the two coexisting phases, we have used three different approaches. Coupleddecoupled $\mathrm{CBMC}^{10}$ can be used without modifications for particle swaps of the semiflexible alkenes (where only the bond lengths are held rigid). However, the aromatic molecules contain larger rigid portions which require special attention. For completely rigid molecules (benzene, toluene, the xylenes, and naphtalene), the CBMC part of the swap move proceeds as follows: (i) randomly select a trial position (or positions, see below) in the insertion box for the first bead of the molecule to be swapped (usually one of the pseudo-atoms in the benzene ring). (ii) calculate the energies and Boltzmann weights for $n_{\text {choice }}$ random orientations of the rigid molecule (keeping the position of the first bead as the origin). (iii) randomly select with a probability proportional to its Boltzmann weight one of the $n_{\text {choice }}$ orientations as the trial position. and (iv) determine $n_{\text {choice }}-1$ orientations and their energies and Boltzmann weights for the trial molecule in the removal box, and $(v)$

accept or reject the biased swap move according to the regular CBMC acceptance rules. ${ }^{22}$ Finally, if the aromatic molecule consists of a rigid ring and semiflexible alkyl chain parts 
(ethylbenzene, propylbenzene, and isopropylbenzene), then the particle swap starts with an orientational-bias insertion of the rigid part of the molecule which is followed by a coupled-decoupled CBMC growth of the semiflexible part.

Five different types of Monte Carlo moves were employed here: translational, rotational. conformational (using coupled-decoupled CBMC), CBMC particle swap, and volume moves. The type of move was selected at random with the fraction of CBMC particle swap and volume moves adjusted to yield roughly one accepted move per 10 Monte Carlo cycles and with the remainder of moves equally divided between translations, rotations, and conformational changes. Computational efficiency was improved by utilizing a biased insertion with 10 trial sites for the first interaction site in a CBMC particle swap, ${ }^{30,31}$ an additional center-of-mass based cutoff which avoids computing unnecessary distances, ${ }^{9}$ and a shorter potential truncation $\left(r_{\mathrm{CBMC}}=5 \AA\right)$ for use during split-energy CBMC moves ${ }^{32}$ which is then corrected to the full potential $\left(r_{\text {cut }}=14 \AA\right)$ with tail corrections in the acceptance rule.

The NVT-GEMC simulations for single-component systems were carried out using 400 and 300 molecules for the alkenes and alkylbenzenes, respectively. For all of these simulations at least 25,000 Monte Carlo cycles were carried out for the equilibration periods and between 50,000 to 200,000 cycles were used for the production runs.

To estimate the critical temperatures $T_{\mathrm{c}}$ and densities $\rho_{\mathrm{c}}$, the density scaling law (with $\beta=0.32)^{33}$ and the law of rectilinear diameters ${ }^{34}$ were used. Normal boiling points $T_{\mathrm{b}}$ were determined from fits of the vapor pressures to the Clausius-Clapeyron equation. No finite-size or crossover effects were considered here for locating the critical point, but we checked that the density differences obey the scaling law over the range of temperatures studied here (which typically range from $0.5 T_{c}$ to $0.9 T_{c}$ ). If more accurate estimates of the critical properties are desired, then one has to use more elaborate simulation techniques. ${ }^{35}$ 
However, one might argue that the approximate nature of the simple united-atom force fields used here, does not justify the additional effort. Furthermore, no significant difference has been found between critical points of linear alkanes determined from GEMC simulations with a protocol similar to that used here ${ }^{9}$ and from grand canonical Monte Carlo simulations with histogram reweighting and mixed-field scaling analysis. ${ }^{36}$

Simulations using the isobaric version of the Gibbs ensemble ${ }^{24}$ were carried out to determine the pressure-composition diagrams for binary systems. The total numbers of molecules used were 400 and 300 for the ethene $/ n$-heptane and benzene $/ n$-pentane simulations, respectively, and the overall mole fractions were adjusted to yield similar numbers of molecules in the two simulation boxes. The equilibration and production periods consisted of at least 25,000 and 50,000 Monte Carlo cycles, respectively. In addition, NVT GEMC simulations were also performed to determine the corresponding saturated vapor pressures of pure benzene and pure $n$-pentane.

\section{Results and Discussion}

A. Vapor-Liquid Phase Equilibria for Alkenes. Following a similar strategy as employed in the development of the TraPPE-UA force field parameters for saturated alkanes, ${ }^{9,10}$ simulations were performed first for ethene to determine suitable LJ parameters for the $\mathrm{CH}_{2}\left(\mathrm{sp}^{2}\right)$ pseudo-atom. The vapor-liquid coexistence curve (VLCC) for ethene calculated using the OPLS-UA force field ${ }^{1}$ is shown in Figure 1. Considering the usually fair performance of this force field for the $n$-alkanes, ${ }^{9}$ the results for ethene are disappointing: in particular, the orthobaric liquid densities and the critical temperature are underestimated by about $23 \%$ and $16 \%$, respectively. It should be noted here, that Jorgenson et al. ${ }^{1}$ did not carry out separate calculations for ethene, but determined the $\mathrm{CH}_{2}\left(\mathrm{sp}_{2}\right)$ parameters from simulations of 1-butene. We carried out GEMC simulations for ethene to determine suitable LJ parameters for the $\mathrm{CH}_{2}\left(\mathrm{sp}^{2}\right)$ group, emphasizing agreement with experimen- 
tal satmated liquid densities and the critical temperature in the fitting procedure. The resulting TraPPE-LA LJ diameter is $0.7 \%$ smaller and LJ well depth is $5.9 \%$ smaller than the corresponding OPLS-UA paramters (see Table 1). The VLCC of ethene calculated for the TraPPE-LA force field is shown in Figure 1 and the comesponding numerical dara are lisied in Table S1. Whereas the experimental saturated liquid densities are reproduced with a mean unsigned error of $0.3 \%$, the saturated vapor densities (and correspondingly also the saturated vapor pressures) are consistently overestimated. Although the normal boiling point is underestimated by about $3 \%$, agreement for the critical temperature and critical density is very good. The deviations from the experimental VLCC are also reflected in the heats of vaporization (not shown), where the TraPPE-UA force field gives satisfactory results at higher temperatures (as should be expected from the good prediction of the critical temperature), but yields too small heats of vaporization at the lower temperatures. It has to be emphasized here, that similar problems were also observed for the saturated alkanes. ${ }^{9,10}$ Thus. we would like to argue that this problem is caused mainly by the oversimplified united-atom representation of the molecular shape and should not be attributed to the neglect of special interactions for the unsaturated bonds. Better agreement with experiment might be obtained by adjusting the separation of the two pseudo-atoms in ethene, that is placing the interaction sites further apart than the length of a $\mathrm{C}=\mathrm{C}$ double bond, as is done in the SET force field for ethene ${ }^{8}$, the Toxvaerd anisotropic united-atom force field ${ }^{37}$ and the Gibbs99 exp-6 force field ${ }^{36}$ for the alkanes, and as has been suggested much earlier from calculations of radial distribution functions for ethane by Byrnes and Sandler. ${ }^{38}$ Thus although placing all pseudo-atom interaction sites at the positions of carbon nuclei, as is done in the OPLS-UA and TraPPE-UA force fields, is the convenient choice. it may not be the optimal choice for a given molecule. Nevertheless, we feel that this choice is still the best option for a general united-atom force field because it greatly enhances the transferability to different molecules, whereas searching for improved pseudo-atom separations will invariably lead to different "bond" lengths for methyl-methyl 
and methyl-methylene ${ }^{36}$ or $\mathrm{CH}_{2}\left(\mathrm{sp}^{2}\right)-\mathrm{CH}_{2}\left(\mathrm{sp}^{2}\right)$ and $\mathrm{CH}_{2}\left(\mathrm{sp}^{2}\right)-\mathrm{CH}\left(\mathrm{sp}^{2}\right)$. Finally: we would expect that using an explicit-hy-drogen representation for the alkenes (which gives a much betrer representation of the molecular shape) one can obtain much better agreement between simulation and experiment albeit at a much higher computational cost. This has been convincingly demonstrated for the linear alkanes. ${ }^{11}$

The other pseudo-atom needed for the modeling of alkenes are the $\mathrm{CH}\left(\mathrm{sp}^{2}\right)$ and $\mathrm{C}\left(\mathrm{sp}^{2}\right)$ groups. Here we performed GEMC calculations for cis-2-butene and 2-methylpropene to determine the LJ parameters for these sites retaining the $\mathrm{CH}_{2}\left(\mathrm{sp}^{2}\right)$ parameters from ethene and the methyl group parameters from the TraPPE-UA force field for the saturated alkanes. ${ }^{9,10}$ Another option would have been to use propene in the fitting procedure, but we decided on cis-2-butene because of its greater abundance of $\mathrm{CH}\left(\mathrm{sp}^{2}\right)$ pseudo-atoms. These molecules were also used by Jorgensen et al. ${ }^{1}$ in the determination of the OPLS-UA force field. The OPLS-UA and TraPPE-UA parameter for these group are listed in Table 1. Since the methyl group parameters of the OPLS-UA and TraPPE-UA force fields differ, the $\mathrm{CH}\left(\mathrm{sp}^{2}\right)$ LJ parameters are necessarily also different; for example, while the methyl group well depth is about $10 \mathrm{~K}$ smaller for the OPLS-UA force field, its $\mathrm{CH}\left(\mathrm{sp}^{2}\right)$ group well depth is about $10 \mathrm{~K}$ larger. The differences for the $\mathrm{C}\left(\mathrm{sp}^{2}\right)$ group are much more pronounced, but it should be noted here that the OPLS-UA force field employs a different set of methy] group parameters when this group is connected to a carbon branch point. ${ }^{1}$ The VLCC of cis-2-butene and 2-methylpropene calculated for the TraPPE-UA force field are shown in Figure 2 (see also Table S1). Again, agreement with experiment for the saturated liquid densities and the critical temperatures is very good, whereas vapor densities and pressures are overestimated and the normal boiling points are underestimated. Calculations for the OPLS-UA force field were not performed, but a comparison of the TraPPE-UA and OPLS-UA LJ diameters demonstrates that the OPLS-UA will yield liquid densities for cis-2-butene that are significantly too low (as has been observed in isobaric-isothermal 
ensemble simulations ${ }^{1}$ ).

The force field parameters for the three si ${ }^{2}$-type psendo-atoms (in conjunction with the previously developed saturated methyl and methylene psendo-atoms ${ }^{9,10}$ ) were then tested for their transferability to other alkenes. VLCC for some $\alpha$-olefines are compared in Figure 1 (see also Table S1) and the corresponding thermodynamics properties are listed in Table 5. Agreement with the experimental data for propene, 1-butene, and 1-octene is satisfactory (good agreement in saturated liquid densities and critical temperatures, but again an overprediction of the saturated vapor pressures). It is rather encouraging that the thermodynamic properties of these three $\alpha$-olefines are reproduced with similar accuracy as those of ethene and cis-2-butene used for the fitting of the $\mathrm{CH}_{2}\left(\mathrm{sp}^{2}\right)$ and $\mathrm{CH}\left(\mathrm{sp}^{2}\right)$ pseudoatoms. This outcome should not have been taken for granted because of the different nature of carbon-carbon $\sigma$ and $\pi$ bonds that might affect the transferability of pseudo-atoms between saturated and unsaturated hydrocarbons. The OPLS-UA force field performs much better for 1-butene than for ethene and propene (and from comparison of these three alkene, one might expect that agreement will worsen for longer a-olefines as it does for longer $n$-alkanes $\left.{ }^{9}\right)$, but 1-butene was used in the OPLS- $L A$ fitting procedure. Performance of the SET force field for 1-octene is comparable to that of the TraPPE-UA force field, but small differences are noticeable which result from the different fitting strategies. Whereas agreement with experimental vapor pressures was part of the development of the SET force field. the TraPPE-UA force field was fitted emphasizing the saturated liquid densities. As has been discussed by Errington and Panagiotopoulos ${ }^{39}$ and by us. ${ }^{11}$ the united-atom representation using $\mathrm{LJ}$ potentials and realistic carbon-carbon bond lengths for the pseudoatom separations is apparently not flexible enough to describe with sufficient accuracy the molecular shape and the temperature- and density-dependences of the cohesive interactions in liquid alkanes or alkenes. Thus compromises have to be made in the development of computationally efficient united-atom force fields. Finally, the results for 1-butene obtained 
for the SET force field ${ }^{6}$ deviate more significantly from experiment than those for 1-octene. This lets us to speculate that modeling $\mathrm{CH}_{3}\left(\mathrm{sp}^{3}\right)$ and $\mathrm{CH}_{2}\left(\mathrm{sp}^{3}\right)$ pseudo-atoms with the same parameters, as is done in the SET force field (see Table 1), restricts the transferability of the parameters to a-olefines of only a certain length range.

Since the $\mathrm{CH}\left(\mathrm{sp}^{2}\right)$ pseudo-atom parameters were fitted for cis-2-butene, it is instrumental to test that these parameters can also be applied to the trans-isomers. The VLCC of trans-2-butene is shown in Figure 2 and its thermodynamic properties are listed in Table 5. Agreement with experiment is very encouraging, in particular. the TraPPE-UA force field predicts correctly that the critical temperatures of the two 2-butene isomers differ by a large amount ( $7 \mathrm{~K}$ in the experiments ${ }^{42}$ versus $9 \mathrm{~K}$ in the simulations), whereas the difference in normal boiling points is small ( $3 \mathrm{~K}$ and $2 \mathrm{~K}$, respectively). The differences in saturated liquid densities are similarly well described (see Table S1). The liquid-phase radial distribution functions (RDFs) for cis- and trans-2-butene are compared in Figure 3. Overall the structural differences between the two isomers are rather small. In particular, the methyl-methyl and $\mathrm{CH}\left(\mathrm{sp}^{2}\right)-\mathrm{CH}\left(\mathrm{sp}^{2}\right) \mathrm{RDF}$ for both isomers are very similar, but more noticeable differences are observed for the methyl-CH( $\left.\mathrm{sp}^{2}\right) \mathrm{RDF}$. Given the small differences in RDFs. it is remarkable that the difference in bulk thermodynamic properties are well reproduced. Finally the VLCC for 1.5-hexadiene was also calculated. Agreement with the rather limited experimental data (orthobaric vapor and liquid densities at low temperatures and normal boiling point) for this molecule is satisfactory and shows that the TraPPE-UA force field can be directly applied to non-conjugated poly-enes. However. one might expect lesser agreement for the special cases of conjugated poly-enes which were not simulated here.

B. Vapor-Liquid Phase Equilibria for Alkylbenzenes. Simulations were carried out first for benzene which is treated in the OPLS-UA and the TraPPE-UA force fields 
as a rigid, planar hexagon containing six identical CH(aro) psendo-atoms. Although the OPLS-UA ${ }^{1}$ force field yields satisfactory orthobaric vajor and liquid densities at $300 \mathrm{~K}$ (very close to the thermodynamic conditions of $T=298.15 \mathrm{~K}$ and $p=101.3 \mathrm{kPa}$ which were used in the fitting procedure for this force field). the agreement with experiment worsen with increasing temperature (see Figure 5), and the OPLS-UA rields a critical temperature that is about $80 \mathrm{~K}$ too high. Thereafter, we tested the OPLS-AA force field ${ }^{2}$ for benzene which uses additional LJ interaction sites at the hydrogen positions and partial charges at all carbon and hydrogen nuclei. Again, agreement at the lower temperatures is satisfactory, but the saturated vapor and liquid densities at higher temperatures are too low. The resulting critical temperature and density are $2.5 \mathrm{~K}$ and $0.043 \mathrm{~g} / \mathrm{mL}$ lower than their experimental counterparts.

VLCC calculations for benzene and toluene were used to determine the TraPPE-UA parameters for the aromatic $\mathrm{CH}($ aro $)$ and $\mathrm{R}-\mathrm{C}$ (aro) groups. The resulting $\mathrm{LJ}$ parameters for $\mathrm{CH}$ (aro) are relatively similar to those for the $\mathrm{CH}\left(\mathrm{sp}^{2}\right)$ pseudo-atoms used for the alkenes, and also the parameters for the $\mathrm{R}-\mathrm{C}(\mathrm{aro})$ and $\mathrm{C}\left(\mathrm{sp}^{2}\right)$ pseudo-atoms are rather close (see Table 1). This observation supports the view that the valence electrons of the $\mathrm{C}-\mathrm{H}$ bonds dominate the polarizability of hydrocarbons. ${ }^{11-13}$ The VLCCs of benzene and toluene are well reproduced by the TraPPE-UA force field. As was also found for the alkanes and alkenes, agreement with experimental saturated liquid densities is very good (less than $1 \%$ deviation over the entire temperature ranges), but vapor pressures and densities are again overpredicted. Although the normal boiling points are underestimated by about $3 \%$, the critical temperatures are very close to their experimental counterparts. Recently, Errington and Panagiotopoulos ${ }^{40}$ proposed a semiflexible united-atom model for benzene using Buckingham exp-6 potential functions. This exp-6 model yields excellent agreement for both vapor and liquid-phase properties, but it is specific to benzene and its transferability to other alkylbenzenes has not yet been tested. 
The center-of-mass and carbon-carbon RDFs for benzene are compared for the (OPLSUA. OPLS-A.A, and TraPPE-UA force fields in Figure 6. Thereas the two mitcol-atom representations give nearly indistinguishable RDFs, those for the OPLS-AA model differ markedly. In particular, the first peak in the center-of-mass RDF is shifted to shorter separations despite the lower liquid density for the OPLS-AA model. In addition. we observe a second and third peak at 9.5 and $11.5 \AA$, respectively; whereas the mited-atom models show only one broad second peak centered around $10 \AA$. In a similar fashion, the carbon-carbon RDF for the OPLS-AA atom model shows more structural features and a shoulder at the smallest separation that is absent for the united-atom models.

The VLCCs of ethylbenzene, propylbenzene, isopropylbenzene, and the three xylene isomers were calculated (see Figures 7 and 8) to evaluate the transferability of the $\mathrm{CH}$ (aro) and $\mathrm{R}-\mathrm{C}$ (aro) pseudo-atoms. The TraPPE-UA force field performs remarkedly well for the ethylbenzene and the two propylbenzene isomers which have very similar saturated liquid densities around $400 \mathrm{~K}$ (see Table S1), but differ substantially in normal boiling points and critical temperatures (see Table 5). Again, we would like to emphasize here that this good agreement should not be taken for granted because these alkylbenzenes involve unlike interactions between aromatic groups, $\mathrm{CH}($ aro $)$ and $\mathrm{R}-\mathrm{C}($ aro $)$, and saturated groups. $\mathrm{CH}_{2}\left(\mathrm{sp}^{3}\right)$ and $\mathrm{CH}\left(\mathrm{sp}^{3}\right)$, that were not considered while fitting the aromatic groups. In particular, the aromatic $\pi$ clouds result in a non-negligible quadrupole moment for the benzene rings that is not treated explicitly here but simply treated in an effective way by the L.J sites on the aromatic rings.

The VLCCs of the three xylene isomers are less well reproduced. Whereas the calculated orthobaric liquid densities are within $1 \%$ of experiment for all other molecules considered here, the results for the xylenes deviate by about $2 \%$ with the largest deviations observed for $m$-xylene at higher temperatures. In addition, the relative ordering of the liquid densities is not predicted correctly. Whereas the experimental liquid densities 
scale as $1.02: 1.00: 0.99$ for $o^{-}$: $m$-, and $p$-xylene, respectively, the simulations yield $1.00: 1.00: 0.98$. In a similar fashion, the experimental critical temperatures and normal boiling points of m- and p-xylene are close, but the TraPPE-UA force field predicts smaller differences between $o$ - and $m$-xylene. An explanation for these problems is not obvious, but one might speculate that the close vicinity of the methyl groups in o-xylene might affect the polarizibility of these groups or the planarity and bond bending angles involving the methyl groups.

Finally. calculations for naphtalene were carried out to obtain the LJ parameters for the $\mathrm{C}$ (aro) pseudo-atom that connects between benzene rings. The calculated orthobaric liquid densities and critical temperature are in good agreement with experiment. ${ }^{42}$ but the calculated boiling temperature is about $5 \%$ too low (experimental vapor densities were not found in the literature). This problem might be attributed to treating an entire naphtalene as a rigid unit, while it is actually somewhat flexible. ${ }^{43}$ This is an issue that should be addressed before extending the calculations to larger aromatic poly-cyles for which experimental VLCC data are not available.

C. Binary Mixtures of Alkanes with Ethene or Benzene. To assess the transferability of the new TraPPE-UA force field for alkenes and alkylbenzene, we carried out isobaric Gibbs ensemble simulations for two binary mixtures. The $433 \mathrm{~K}$ isotherm for the mixture of (supercritical) ethene and $n$-heptane is shown in Figure 9 and the corresponding numerical results are listed in Table S3. Agreement with experiment is very satisfactory and of similar quality as TraPPE-UA calculations for the mixture of (supercritical) ethane and $n$-heptane. Thus the unlike interactions between the saturated and vinylic pseudoatoms are well accounted for and we apparently do not require special $\pi-\pi$ interactions to treat alkenes and their mixtures with alkanes with satisfactory accuracy. Of course. we should not ignore that the saturated vapor pressures calculated with the TraPPE-LA 
for both pure ethene and pure $n$-heptane are slightly too high, but these small shifts are marked by the large pressure scale of the phase diagram for the supercritical mixtmere. Analysis of RDFs and their number integrals (not shown) supports a picture of the supercritical phase with random mixing of the supercritical ethene solvent and the n-heptane solute, that is there is no evidence for clustering of solvent molecules around a solute. In general, this supercritical solvent/solute system behaves rery similar to the related binary mixture of ethane and $n$-heptane which has been discussed in more detail in a previous publication. ${ }^{44}$

The pressure-composition diagram for the mixture of $n$-pentane and benzene at $T=$ $318.15 \mathrm{~K}$ is shown in Figure 10. Since the vapor pressures of both $n$-pentane and benzene are overestimated by about $50 \%$ at this relatively low temperature and because both molecule have relatively similar vapor pressures, the calculated pressure-compositions diagram differs substantially from experiment. In fact, the calculated phase diagram is close to its experimental counterpart at $10 \mathrm{~K}$ higher temperature, as should be expected from the offsets in normal boiling points (see Table 5). Nevertheless, from a plot of the vapor- versus liquid-phase mole fraction of $n$-pentane (see Figure 10): it is apparent the TraPPE-U.A force field yields much better predictions of the separation factors. The separation factors determined from Raoult's law for ideal binary mixtures using either the experimental or the calculated pure-phase vapor pressures are also shown in Figure 10. As can be seen. the simulations predict correctly positive deviations from Raoult's law (higher vapor-phase mole fractions of $n$-pentane) at low $n$-pentane concentrations in the liquid phase and negative deviations from Raoult's law at high $n$-pentane concentrations. Recently; Lisal et al. ${ }^{45}$ proposed a simulation procedure for multi-component phase equilibria which involves rescaling of the chemical potentials of the constituents to account for differences in the experimental and calculated vapor pressures of the pure phases. Clearly, this procedure could also be used here to improve agreement with experiment. However, we would like 
to refrain from such re-scaling procedures which require knowledge of experimental quantities and prefer to use more accumate. but more expensive all-atom and/or polarizable

models to improve the accuracy of the predictions. ${ }^{11.46}$ Finally, analysis of the number integrals of the RDFs shows random mixing for the n-pentane/benzene mixture over the entire range of pressures or compositions (see Figure 11).

\section{Conclusions}

The TraPPE-UA force field is extended to linear and branched alkenes and alkylbenzenes by the introduction of six new pseudo-atoms. The performance of the TraPPE-UA force field for the prediction of thermophysical properties is in general very satisfactory. but the main shortcoming of this simple united-atom force field remains the slight overprediction of saturated vapor pressures which was also observed for saturated alkanes. ${ }^{9.10}$ The TraPPE-LA pseudo-atoms are transferable from one molecule type to another using the standard Lorentz-Berthelot combining rules for Lennard-Jones interactions of unlike pseudo-atoms. Whereas the popular OPLS-LA force field clearly does not perform with the same accuracy as the TraPPE- ${ }^{-} A$ force field. the performance of the SET force field is comparable, but some limits of the transferability of the SET parameters are evident. The improved performance of the TraPPE-UA force field does not come at higher computational costs nor does it require the use of a larger number of pseudo-atoms (or parameters). In fact, to describe alkanes and alkenes the OPLS-LA force field uses 11 different pseudo-atoms, compared to 6 and 7 pseudo-atoms for the TraPPE-UA and SET force fields (where the latter is limited to $\alpha$-olefines). In addition, the TraPPE-UA force field is also parametrized for alkylbenzenes and for poly-cylic aromatic systems, whereas the required pseudo-atom parameters are missing in the OPLS-UA force field. Finally, the TraPPE-UA force field was also used to calculate the pressure-composition diagrams of two binary mixtures and the results are promising without requiring any special binary 
mixing parameters.

Acknowledgments. Financial support from the National Science Foundation (CTS-9813601), an Alfred P. Sloan Research Fellowship, and a Department of Energy Computational Science Graduate Fellowship (MGM) is gratefully acknowledged. Part of the computer resources were provided by the Minnesota Supercomputing Institute.

Supporting Information Available: The numerical values of the saturated vapor pressures and coexistence densities for all single-component systems are listed in Tables S1 (alkenes) and S2 (alkylbenzenes). The numerical values of the rapor and liquid phase compositions for the binary mixtures are listed in Table S3. 


\section{References}

[1] Jorgensen. W. L.; Madura, J. D.; Swenson, C. J. J. Am. Chem. Soc. 1984. 106. 663 S.

[2] Jorgensen, W. L.; Severance, D. L. J. Am. Chem. Soc. 1990, 112, 4768.

[3] Jorgensen. W. L.; Maxwell, D. S.; Tirado-Rives, J. J. Am. Chem. Soc. 1996. 11 s. 11225 .

[4] Siepmann, J. I.; Karaborni, S.; Smit, B. Nature 1993, 365, 330.

[5] Martin, M. G.; Siepmann, J. I. J. Am. Chem. Soc. 1997, 119, 8921.

[6] Spyriouni, T.; Economou, I. G.; Theodorou. D. ‥ J. Am. Chem. Soc. 1999. 1?1. 3407.

[7] Dodd, L. R.; Theodorou, D. N. Adv. Pol. Sci. 1994. 116, 249.

[8] Spyriouni. T.; Economou, I. G.; Theodorou, D. N. Phys. Rev. Lett. 1998, 80, 4466.

[9] Martin, M. G.: Siepmann, J. I. J. Phys. Chem. B. 1998. 102. 2569.

[10] Martin, M. G.: Siepmann, J. I. J. Phys. Chem. B. 1999. 103, 4508.

[11] Chen, B.: Siepmann, J. I. J. Phys. Chem. B. 1999. 103, 5370.

[12] Amos, A. T.: Crispin, R. J. in Theoretical Chemistry: Advances and Perspectives. eds. Eyring, H.: Henderson, D. Vol. 2, pp. 2. Academic. New York, 1976.

[13] Kaplan, I. G. Theory of Molecular Interactions, Translation eds. Fraga, S.; Klobukowski, M. Elsevier Science Publishers B.V., 1986.

[14] Chen, B.; Stubbs, J. M.; Potoff, J. J.; Siepmann, J. I. in preparation.

[15] Lorentz, H.A. Annalen Phys. 1881, 12, 127. 
[16: Berthelot. D. C. r. hebd. Séanc. Acad. Sci, Paris 1898, 126, 1703.

[17. Wood. IT. W.: Parker, F. R. J. Chem. Phys. 1955. 27, 720.

[18: Allen, M. P.: Tildesley, D. J. Computer Simulation of Liquids (Oxford University Press, Oxford. 1987).

[19] Van der Ploeg. P.: Berendsen, A. J. Chem. Phys. 1978, 76. 3271.

[20 Cornell. IV. D.; Cieplak. P.; Bayly; C.; Gould, I. R.: Merz; K. M.; Ferguson, D. M.: Spellmeyer, D. C.: Fox, T.; Caldwell, J. W.; Kollman. P. A. J. Am. Chem. Soc. 1995. 117.5179 .

[21: PC-Model for Windows. version 5.13; Serena Software.

[22. Mooij, G. C. A. M.; Frenkel, D.; Smit, B.; J. Phys.: Cond. Matt. 1992, 4. L255.

[23] Panagiotopoulos, A. Z. Mol. Phys. 1987, 61, 813.

[24] Panagiotopoulos, A. Z.: Quirke, N.: Stapleton, M.: Tildesley, D. J. Mol. Phys. 1988. $63.52 \pi$.

[25. Snit, B.: de Smedt, P.: Frenkel, D. Mol. Phys. 1989, 68, 931.

[26] Siepmann, J. I. Mol. Phys. 1990, 70, 1145.

[27] Siepmann, J. I.: Frenkel. D. Mol. Phys. 1992, 75. 59.

[28] Frenkel, D.; Mooij, G. C. A. M.; Smit, B. J. Phys.: Cond. Matt. 1992, 4. 3053.

[29] de Pablo, J. J.; Laso, M.; Suter, U. W. J. Chem. Phys. 1992, 96, 2395.

[30] Esselink, K.; Loyens, L. D. J. C.; Smit, B. Phys. Rev. E 1995, 51, 1560.

[31] Mackie, A. D.: Tavitian, B.; Boutin, A.; Fuchs, A. H. Mol. Sim. 1997, 19, 1. 
[32] Vlugt, T. J. H.; Martin, M. G.: Smit, B.; Siepmann, J. I.; Krishna, R. Mol. Phys. 1998, 94,727 .

[33] Rowlinson, J. S.; Widom, B. Molecular Theory of Capillarity; Oxford University Press: New York, 1989; p 261.

[34] Rowlinson, J. S.; Widom, B. Liquids and Liquid Mixtures, 3rd ed.; Butterworth: London, 1982; pp 70-75.

[35] Potoff, J. J.; Panagiotopoulos, A. Z. J. Chem. Phys. 1998, 109, 10914.

[36] Errington, J. R.; Panagiotopoulos, A. Z. J. Phys. Chem. B 1999, 103, 6314.

[37] Toxvaerd, S. J. Chem. Phys. 1990, 93, 4290.

[38] Byrnes, J. M.; Sandler, S. I. J. Chem. Phys. 1984, 80, 881.

[39] Errington, J. R.; Panagiotopoulos, A. Z. J. Chem. Phys. 1998, 109, 1093.

[40] Panagiotopoulos, A. Z.; Errington, J. R., J. Chem. Phys. 1999, 111, 9731.

[41] Smith, B. D.; Srivastava, R.; Thermodynamic Data for Pure Compounds: Part A Hydrocarbons and Ketones, Elsevier: Amsterdam, 1986.

[42] Yaws, C. L.; Thermodynamic and Physical Property Data, Gulf Publishing Company: Houston, 1992.

[43] Lipkowitz, K. B.; Peterson, M. A. J. Comput. Chem. 1994, 14, 121.

[4] Martin. M. G.; Chen, B.; Siepmann. J. I. J. Phys. Chem. B 2000, 104, 2415.

[45] Lísal, M.; Smith, W. R.; Nezbeda. I. J. Phys. Chem. B 1999, 103, 10496.

[46] Chen. B.; Xing, J.; Siepmann, J. I. J. Phys. Chem. B 2000, 104, 2391.

[47] Knapp, H.; Doring, R.; Oellrich, L.; Prausnitz, J. M. Vapor-Liquid Equilibria for Mixtures of Low Boiling Substances; Schon and Wetzel: Frankfurt/Main, 1982. 


\section{FIGURE CAPTIONS}

Figure 1. Vapor-liquid coexistence curves for ethene, propene, 1-butene. and 1-octene. Calculated coexistence densities and extrapolated critical points for the TraPPE-UA. OPLS-UA, ${ }^{1}$ and SET $^{6}$ force fields are shown as open circles, squares. and diamonds. respectively. Experimental coexistence data and critical points are depicted by solid lines and stars. ${ }^{41}$

Figure 2. Vapor-liquid coexistence curves for 2-methylpropene. cis-2-butene, and trans-2-butene. Calculated coexistence densities and extrapolated critical points for the TraPPE-UA force field are shown as open circles, squares, and diamonds, respectively. Experimental coexistence data and critical points are depicted as solid lines and a plus for 2-methylpropene, dotted lines and a star for cis-2-butene, and dashed lines and an inverted triangle for trans-2-butene. ${ }^{41}$

Figure 3. Intermolecular segment-segment radial distribution functions for cis-2-butene at $T=322 \mathrm{~K}$ (solid lines) and trans-2-butene at $T=323 \mathrm{~K}$ (dashed lines). The radial distribution functions are shown for $\mathrm{CH}-\mathrm{CH}, \mathrm{CH}-\mathrm{CH}_{3}$ (displaced vertically by 1 unit). and $\mathrm{CH}_{3}-\mathrm{CH}_{3}$ pairs (displace vertically by 2 units).

Figure 4. Vapor-liquid coexistence curves for 1,j-hexadiene. Calculated coexistence densities and extrapolated critical point are shown as open circles. Experimental orthobaric liquid densities are depicted by the solid line.

Figure 5. Vapor-liquid coexistence curves for benzene, toluene, and naphtalene. Calculated coexistence densities and extrapolated critical points for the TraPPE- $\mathrm{C} A$. OPLS$\mathrm{UA},{ }^{1}$ and OPLS-AA ${ }^{2}$ force fields are shown as open circles, squares, and diamonds. respectively. Experimental coexistence data and critical points are depicted by solid lines and the star. ${ }^{41}$ 
Figure 6. Center-of-mass and carbon-carbon (displaced vertically by 1 mit) radial distribution functions for benzene at $T=400 \mathrm{~K}$. Solid. dotted. and dianhed line styles are used to depict the results for the TraPPE-UA. OPLS- $A$ A and OPLS-A force fieds.

Figure 7. Vapor-liquid coexistence curves for ethylbenzene, proprlbenzene, and isopropylbenzene. Calculated coexistence densities and extrapolated critical points are shown as open circles, squares, and diamonds, respectively. Experimental coexistence data and critical points ${ }^{42}$ are depicted as solid lines and a star for ethylbenzene, dotter lines and a plus for propylbenzene, and dashed lines and an inverted triangle for isopropylbenzene.

Figure 8. Vapor-liquid coexistence curves for $0-m$ - and $p$-xylene. Calculated coexistence densities and extrapolated critical points are shown as open circies. squares. and diamonds, respectively. Experimental coexistence data and critical points ${ }^{41}$ are depicted as solid lines and a star for o-xylene, dotted lines and a plus for m-xylene. and dashed lines and an inverted triangle for $p$-xylene.

Figure 9. Pressure-composition diagram for the $433 \mathrm{~K}$ isotherm of the binary mixture consisting of supercritical ethene and n-heptane calculated using the TraPPE-C A force field (diamonds). The solid line represents the experimental results. ${ }^{i}{ }^{i}$

. Figure 10. Pressure-composition (top) and $x_{1} / y_{1}$ (bottom) diagrams for the 318.15 $\mathrm{K}$ isotherm of the binary mixture consisting of $n$-pentane and benzene calculated using the TraPPE-UA force field (circles). The solid lines represents the experinentil resils. ${ }^{\circ}$ The $x_{1} / y_{1}$ ralues determined from Raoult's law for ideal binary mixtures using either the experimental or the calculated pure-phase vapor pressures are depicted by the dashed line and squares; respectively.

Figure 11. Number integrals calculated from center-of-mass radial distribution functions for the liquid phases of the binary $n$-pentane/benzene mixture at $t=318.15 \mathrm{~K}$ 
and $p=1.76 \mathrm{MPa}$ (top) and $p=0.898 \mathrm{MPa}$ (bottom). Number integrals are shorn for $n$-pentane surrounding $n$-pentane (solid lines), n-pentane surrounding benzene (squares), benzene surrounding benzene (dashed lines), and benzene surrounding $n$-pentane (circles). 
Tables

Table 1: Comparison of the Lennard-Jones Parameters for the OPLS-UA, ${ }^{1}$ SET, ${ }^{6-8}$ and TraPPE-UA Force Fields

\begin{tabular}{|c|c|c|c|c|c|c|c|}
\hline \multirow[b]{2}{*}{ pseudo-atom } & \multirow[b]{2}{*}{ molecule } & \multicolumn{2}{|c|}{ OPLS-UA } & \multicolumn{2}{|c|}{ SET } & \multicolumn{2}{|c|}{ TraPPE-UA } \\
\hline & & $\sigma[\AA]$ & $\epsilon / k_{B}[K]$ & $\sigma[A]$ & $\epsilon / k_{\mathrm{B}}[\mathrm{K}]$ & $\sigma[\AA]$ & $\epsilon / k_{\mathrm{B}}[\mathrm{K}]$ \\
\hline $\mathrm{CH}_{3}(\mathrm{sp} 3)$ & ethane & 3.775 & 104.1 & 3.64 & 112.2 & 3.75 & 98.0 \\
\hline $\mathrm{CH}_{3}(\mathrm{sp} 3)$ & $n$-alkane & 3.905 & 88.1 & 3.94 & 49.32 & 3.75 & 98.0 \\
\hline $\mathrm{CH}_{3}(\mathrm{sp} 3)$ & isobutane & 3.910 & 80.5 & $N / A$ & $\mathrm{~N} / \mathrm{A}$ & 3.75 & 98.0 \\
\hline $\mathrm{CH}_{3}(\mathrm{sp} 3)$ & 1-alkene & 3.905 & 88.1 & 3.915 & 47.66 & 3.75 & 98.0 \\
\hline $\mathrm{CH}_{2}(\mathrm{sp} 3)$ & $n$-alkane & 3.905 & 59.4 & 3.94 & 49.32 & 3.95 & 46.0 \\
\hline $\mathrm{CH}_{2}(\mathrm{sp} 3)$ & 1-alkene & 3.905 & 59.4 & 3.915 & 47.66 & 3.95 & 46.0 \\
\hline $\mathrm{CH}(\mathrm{sp} 3)$ & isobutane & 3.850 & 40.3 & $N / A$ & $\mathrm{~N} / \mathrm{A}$ & 4.65 & 10.0 \\
\hline $\mathrm{CH}_{2}(\mathrm{sp} 2)$ & ethene & 3.850 & 78.5 & 3.63 & 91.4 & 3.675 & 85.0 \\
\hline $\mathrm{CH}_{2}(\mathrm{sp} 2)$ & 1-alkene & 3.850 & 78.5 & 3.905 & 89.93 & 3.675 & 85.0 \\
\hline $\mathrm{CH}(\mathrm{sp} 2)$ & 2-alkene & 3.800 & 57.9 & 3.915 & 81.89 & 3.73 & 47.0 \\
\hline$C(\operatorname{sp} 2)$ & isobutene & 3.750 & 52.8 & $N / A$ & $N / A$ & 3.85 & 20.0 \\
\hline $\mathrm{CH}$ (aro) & benzene & 3.750 & 55.4 & $N / A$ & $\mathrm{~N} / \mathrm{A}$ & 3.695 & 50.5 \\
\hline $\mathrm{R}-\mathrm{C}$ (aro) & alkylbenzene & $N / A$ & $N / A$ & $N / A$ & $N / A$ & 3.88 & 21.0 \\
\hline $\mathrm{C}($ aro link) & napthalene & $\mathrm{N} / \mathrm{A}$ & $N / A$ & $N / A$ & $N / A$ & 3.70 & 30.0 \\
\hline
\end{tabular}


Table 2: Bond Lengths for the TraPPE-UA Force Field

\begin{tabular}{lc}
\hline bond type & $\left.r_{0} \cdot \dot{-}\right]$ \\
\hline $\mathrm{CH}_{x}-\mathrm{CH}_{y}$ & 1.54 \\
$\mathrm{CH}_{x}$ (aro) $-\mathrm{CH}_{y}$ (aro) & 1.40 \\
$\mathrm{CH}_{x}=\mathrm{CH}_{y}$ & 1.33 \\
\hline
\end{tabular}

Table 3: Equilibrium Bond Angles and Force Constants for the TraPPE-UA Force Field

\begin{tabular}{lcc}
\hline bend type & $\theta_{0} \cdot$ deg $]$ & $k_{\theta} / k_{\mathrm{B}}[\mathrm{K}]$ \\
\hline $\mathrm{CH}_{x}-\mathrm{CH}_{2}\left(\mathrm{sp}^{3}\right)-\mathrm{CH}_{y}$ & 114 & 62500 \\
$\mathrm{CH}_{x}-\mathrm{CH}\left(\mathrm{sp}^{3}\right)-\mathrm{CH}_{y}$ & 112 & 62500 \\
$\mathrm{CH}_{x}\left(\mathrm{sp}^{2}\right)=\mathrm{CH}\left(\mathrm{sp}^{2}\right)-\mathrm{CH}_{y}$ & 119.7 & 70420 \\
$\mathrm{CH}_{x}\left(\mathrm{sp}^{2}\right)=\mathrm{C}\left(\mathrm{sp}^{2}\right)-\mathrm{CH}_{y}$ & 119.7 & 70420 \\
$\mathrm{CH}_{x}-\mathrm{C}\left(\mathrm{sp}^{2}\right)-\mathrm{CH}_{y}$ & 119.7 & 70420 \\
$\mathrm{CH}_{x}$ (aro $)-\mathrm{CH}_{y}(\operatorname{aro})-\mathrm{CH}_{z}$ & 120 & rigid \\
$\mathrm{CH}_{x}($ aro $)-\mathrm{CH}_{y}(\operatorname{aro})-\mathrm{CH}_{z}(\operatorname{aro})$ & 120 & rigid \\
\hline
\end{tabular}


Table 4: Torsional Potential Parameters for the TraPPE-UA Force Field

\begin{tabular}{lcccc}
\hline torsion type & $c_{0} / k_{\mathrm{B}}[\mathrm{K}]$ & $c_{1} / k_{\mathrm{B}}[\mathrm{K}]$ & $c_{2} / k_{\mathrm{B}}[\mathrm{K}]$ & $c_{3} / k_{\mathrm{B}}[\mathrm{K}]$ \\
\hline $\mathrm{CH}_{x}-\mathrm{CH}_{2}-\mathrm{CH}_{2}-\mathrm{CH}_{y}$ & 0.0 & 355.03 & -68.19 & 791.32 \\
$\mathrm{CH}_{x}-\mathrm{CH}_{2}-\mathrm{CH}=\mathrm{CH}_{y}$ & 688.5 & 86.36 & -109.77 & -282.24 \\
\hline & \multicolumn{2}{c}{$d_{0}[\mathrm{~K}]$} & $\phi_{0}[\mathrm{rad}]$ \\
\hline $\mathrm{CH}_{x}-\mathrm{CH}=\mathrm{CH}-\mathrm{CH}_{y} \quad($ cis $)$ & 12400 & $\pi$ \\
$\mathrm{CH}_{x}-\mathrm{CH}=\mathrm{CH}-\mathrm{CH}_{y} \quad($ trans $)$ & 13400 & 0 \\
\hline & $e_{0}[\mathrm{~K}]$ & \multicolumn{2}{c}{$e_{1}[\mathrm{rad}]$} \\
\hline $\mathrm{CH}_{x}($ aro $)-\mathrm{C}($ aro $)-\mathrm{CH}_{2}-\mathrm{CH}_{x}$ & 131 & $\pi$ \\
$\mathrm{CH}_{x}($ aro $)-\mathrm{C}($ aro $)-\mathrm{CH}\left(\mathrm{sp}^{2}\right)-\mathrm{CH}_{x}$ & 167 & \multicolumn{2}{c}{$5 \pi / 3$} \\
\hline
\end{tabular}


Table 5: Comparison of Thermodynamic Properties Calculated for the TraPPE-UA Force Field and Neasured Experimentally ${ }^{41,42}$

\begin{tabular}{lcccccc}
\hline & \multicolumn{2}{c}{$T_{c}[\mathrm{~K}]$} & \multicolumn{2}{c}{$\rho_{c}[\mathrm{~g} / \mathrm{mL}]$} & \multicolumn{2}{c}{$T_{b}[\mathrm{~K}]$} \\
\hline Molecule & calcd & exptl & calcd & exptl & calcd & exptl \\
\hline ethene & 283 & 282.3 & 0.215 & 0.214 & 164 & 169.4 \\
propene & 362 & 365.6 & 0.230 & 0.223 & 217 & 225.4 \\
1-butene & 414 & 419.6 & 0.241 & 0.234 & 256 & 266.9 \\
2-methylpropene & 418 & 417.9 & 0.237 & 0.235 & 258 & 266.3 \\
cis-2-butene & 435 & 435.6 & 0.246 & 0.240 & 264 & 276.9 \\
trans-2-butene & 426 & 428.6 & 0.238 & 0.236 & 262 & 274.0 \\
1-octene & 567 & $\mathrm{~N} / \mathrm{A}$ & 0.638 & $\mathrm{~N} / \mathrm{A}$ & 383 & 394.4 \\
1,5-hexadiene & 496 & $\mathrm{~N} / \mathrm{A}$ & 0.251 & $\mathrm{~N} / \mathrm{A}$ & 329 & 332.7 \\
benzene & 565 & 562.2 & 0.304 & 0.302 & 341 & 353.2 \\
toluene & 594 & 594.0 & 0.298 & 0.309 & 370 & 383.8 \\
ethylbenzene & 620 & 617.2 & 0.287 & 0.284 & 394 & 409.4 \\
propylbenzene & 647 & 638.3 & 0.284 & 0.273 & 424 & 432.4 \\
isopropylbenzene & 643 & 631.1 & 0.276 & 0.280 & 423 & 425.6 \\
o-xylene & 633 & 630.3 & 0.289 & 0.288 & 400 & 417.6 \\
m-xylene & 627 & 617.1 & 0.297 & 0.284 & 400 & 412.3 \\
p-xylene & 617 & 616.2 & 0.283 & 0.281 & 396 & 411.5 \\
napthalene & 750 & 747.8 & 0.312 & 0.314 & 465 & 491.1 \\
\hline & & & & & &
\end{tabular}


Table S1: Numerical Results of the Gibbs-Ensemble Simulations for Alkenes Using the TraPPE-UA Force Field; Experimental Results taken from Refs. 21 and 22

\begin{tabular}{|c|c|c|c|c|c|c|c|}
\hline \multirow[t]{2}{*}{ molecule } & \multirow[t]{2}{*}{$T[\mathrm{~K}]$} & \multicolumn{2}{|c|}{$P[\mathrm{kPa}]$} & \multicolumn{2}{|c|}{$\rho_{\mathrm{liq}}[\mathrm{g} / \mathrm{mL}]$} & \multicolumn{2}{|c|}{$\rho_{\text {vap }}[\mathrm{g} / \mathrm{mL}]$} \\
\hline & & calcd & exptl & calcd & exptl & calcd & exptl \\
\hline \multirow[t]{6}{*}{ ethene } & 144 & 27 & 16.8 & 0.602 & 0.603 & 0.00065 & 0.00040 \\
\hline & 164 & 95 & 72.8 & 0.575 & 0.575 & 0.0020 & 0.0015 \\
\hline & 184 & 280 & 222 & 0.545 & 0.546 & 0.0056 & 0.0043 \\
\hline & 205 & 690 & 556 & 0.513 & 0.513 & 0.0127 & 0.0103 \\
\hline & 225 & 1280 & 1128 & 0.480 & 0.476 & 0.0263 & 0.0206 \\
\hline & 245 & 2390 & 2038 & 0.436 & 0.434 & 0.0447 & 0.0385 \\
\hline \multirow[t]{5}{*}{ propene } & 182 & 16 & 8.1 & 0.660 & 0.661 & 0.00046 & 0.00023 \\
\hline & 213 & 88 & 55.4 & 0.624 & 0.624 & 0.0022 & 0.0014 \\
\hline & 245 & 310 & 228 & 0.582 & 0.584 & 0.0069 & 0.0050 \\
\hline & 276 & 760 & 637 & 0.539 & 0.540 & 0.0160 & 0.0134 \\
\hline & 308 & 1870 & 1467 & 0.485 & 0.487 & 0.0410 & 0.0314 \\
\hline \multirow[t]{6}{*}{ 1-butene } & 245 & 64 & 39.8 & 0.648 & 0.650 & 0.0018 & 0.0011 \\
\hline & 271 & 190 & 119 & 0.618 & 0.620 & 0.0049 & 0.0031 \\
\hline & 296 & 400 & 278 & 0.588 & 0.589 & 0.0100 & 0.0069 \\
\hline & 322 & 770 & 579 & 0.552 & 0.554 & 0.0188 & 0.0141 \\
\hline & 347 & 1320 & 1047 & 0.516 & 0.516 & 0.0324 & 0.0256 \\
\hline & 373 & 2300 & 1784 & 0.467 & 0.470 & 0.0617 & 0.0458 \\
\hline \multirow[t]{4}{*}{ 1-octene } & 346 & 33 & 20.7 & 0.676 & 0.670 & 0.0013 & 0.0008 \\
\hline & 393 & 130 & 97.0 & 0.630 & 0.627 & 0.0048 & 0.00352 \\
\hline & 441 & 390 & $\mathrm{~N} / \mathrm{A}$ & 0.580 & 0.580 & 0.0134 & $N / A$ \\
\hline & 494 & 1120 & $N / A$ & 0.512 & 0.521 & 0.0413 & $\mathrm{~N} / \mathrm{A}$ \\
\hline
\end{tabular}


Table S1: Continued

\begin{tabular}{lccccccc}
\hline molecule & $T[\mathrm{~K}]$ & \multicolumn{2}{c}{$P[\mathrm{kPa}]$} & \multicolumn{2}{c}{$\rho_{\text {liq }}[\mathrm{g} / \mathrm{mL}]$} & \multicolumn{2}{c}{$\rho_{\text {vap }}[\mathrm{g} / \mathrm{mL}]$} \\
& & calcd & exptl & calcd & exptl & calcd & exptl \\
\hline 2-methylpropene & 253 & 81 & 58.7 & 0.642 & 0.642 & 0.0022 & 0.0016 \\
& 274 & 190 & 136 & 0.618 & 0.618 & 0.0049 & 0.0035 \\
& 299 & 410 & 310 & 0.586 & 0.588 & 0.0101 & 0.0077 \\
& 329 & 820 & 701 & 0.545 & 0.548 & 0.0195 & 0.0170 \\
& 350 & 1360 & 1138 & 0.514 & 0.516 & 0.0331 & 0.0280 \\
\hline cis-2-butene & 245 & 47 & 24.9 & 0.676 & 0.675 & 0.0013 & 0.0007 \\
& 295 & 290 & 193 & 0.620 & 0.620 & 0.0072 & 0.0047 \\
& 322 & 560 & N/A & 0.587 & 0.587 & 0.0013 & N/A \\
& 373 & 1840 & N/A & 0.513 & N/A & 0.0453 & N/A \\
\hline trans-2-butene & 247 & 58 & 31.7 & 0.651 & 0.655 & 0.0016 & 0.0009 \\
& 272 & 145 & 93.7 & 0.623 & 0.628 & 0.0038 & 0.0024 \\
& 323 & 670 & 480 & 0.562 & 0.568 & 0.016 & 0.011 \\
& 348 & 1197 & 888 & 0.525 & 533 & 0.029 & 0.021 \\
\hline 1.5-hexadiene & 289 & 21 & 20.2 & 0.702 & 0.697 & 0.00073 & 0.00069 \\
& 324 & 90 & 76.5 & 0.669 & 0.663 & 0.0028 & 0.0023 \\
& 350 & 180 & N/A & 0.640 & N/A & 0.0054 & N/A \\
& 400 & 660 & N/A & 0.587 & N/A & 0.0194 & N/A \\
& 450 & 1885 & N/A & 0.488 & N/A & 0.0594 & N/A \\
\hline
\end{tabular}


Table S2: Numerical Results of the Gibbs-Ensemble Simulations for Alkylbenzenes Using the TraPPE-UA Force Field; Experimental Results taken from Refs. 20 and 21

\begin{tabular}{lccccccc}
\hline molecule & $T[\mathrm{~K}]$ & \multicolumn{2}{c}{$P[\mathrm{kPa}]$} & \multicolumn{2}{c}{$\rho_{\text {liq }}[\mathrm{g} / \mathrm{mL}]$} & \multicolumn{2}{c}{$\rho_{\text {vap }}[\mathrm{g} / \mathrm{mL}]$} \\
\hline benzene & & calcd & exptl & calcd & exptl & calcd & exptl \\
& 300 & 25 & 14.0 & 0.866 & 0.872 & 0.00078 & 0.00044 \\
& 350 & 130 & 91.7 & 0.814 & 0.818 & 0.0037 & 0.0025 \\
& 400 & 480 & 352 & 0.760 & 0.760 & 0.0124 & 0.0090 \\
& 450 & 1130 & 970 & 0.692 & 0.694 & 0.0280 & 0.0245 \\
& 500 & 2400 & 2164 & 0.612 & 0.610 & 0.0616 & 0.0578 \\
toluene & 525 & 3270 & 3057 & 0.550 & 0.552 & 0.0869 & 0.0898 \\
\hline & 400 & 220 & 155 & 0.757 & 0.761 & 0.0065 & 0.0045 \\
& 450 & 600 & 483 & 0.704 & 0.706 & 0.0167 & 0.014 \\
& 500 & 1460 & 1166 & 0.638 & 0.639 & 0.0416 & 0.033 \\
& 530 & 2130 & 1832 & 0.587 & 0.586 & 0.0623 & 0.0545 \\
& 560 & 3060 & 2694 & 0.522 & $\mathrm{~N} / \mathrm{A}$ & 0.0965 & 0.0923 \\
\hline ethylbenzene & 397 & 101 & 71.9 & 0.770 & 0.771 & 0.0035 & 0.0025 \\
& 447 & 380 & 253 & 0.718 & 0.721 & 0.0100 & 0.0750 \\
& 497 & 870 & 669 & 0.659 & $\mathrm{~N} / \mathrm{A}$ & 0.0239 & 0.0205 \\
& 580 & 2532 & 2292 & 0.528 & $\mathrm{~N} / \mathrm{A}$ & 0.0851 & 0.0834 \\
\hline propylbenzene & 350 & 10.0 & 6.72 & 0.809 & 0.814 & 0.00041 & 0.00027 \\
& 400 & 53 & 40.6 & 0.765 & 0.769 & 0.0020 & 0.00154 \\
& 450 & 205 & $\mathrm{~N} / \mathrm{A}$ & 0.726 & $\mathrm{~N} / \mathrm{A}$ & 0.0076 & $\mathrm{~N} / \mathrm{A}$ \\
& 500 & 540 & $\mathrm{~N} / \mathrm{A}$ & 0.672 & $\mathrm{~N} / \mathrm{A}$ & 0.0177 & $\mathrm{~N} / \mathrm{A}$ \\
& 550 & 1150 & $\mathrm{~N} / \mathrm{A}$ & 0.596 & $\mathrm{~N} / \mathrm{A}$ & 0.0389 & $\mathrm{~N} / \mathrm{A}$ \\
& 2106 & $\mathrm{~N} / \mathrm{A}$ & 0.522 & $\mathrm{~N} / \mathrm{A}$ & 0.0777 & $\mathrm{~N} / \mathrm{A}$ \\
\hline
\end{tabular}


Table S2: Continued

\begin{tabular}{|c|c|c|c|c|c|c|c|}
\hline \multirow[t]{2}{*}{ molecule } & \multirow[t]{2}{*}{$T[\mathrm{~K}]$} & \multicolumn{2}{|c|}{$P[\mathrm{kPa}]$} & \multicolumn{2}{|c|}{$\rho_{\mathrm{liq}}[\mathrm{g} / \mathrm{mL}]$} & \multicolumn{2}{|c|}{$\rho_{\text {vap }}[\mathrm{g} / \mathrm{mL}]$} \\
\hline & & calcd & exptl & calcd & exptl & calcd & exptl \\
\hline \multirow[t]{5}{*}{ isopropylbenzene } & 400 & 59 & 49.7 & 0.769 & 0.767 & 0.0022 & $0.0018 \bar{i}$ \\
\hline & 450 & 164 & N/A & 0.715 & 0.718 & 0.0055 & $\mathrm{~N} / \mathrm{A}$ \\
\hline & 500 & 530 & N/A & 0.669 & N/A & 0.0176 & $\mathrm{~N} / \mathrm{A}$ \\
\hline & 550 & 1242 & N/A & 0.608 & $\mathrm{~N} / \mathrm{A}$ & 0.0430 & $\mathrm{~N} / \mathrm{A}$ \\
\hline & 580 & 1872 & $\mathrm{~N} / \mathrm{A}$ & 0.566 & $\mathrm{~N} / \mathrm{A}$ & 0.0701 & N/A \\
\hline \multirow[t]{5}{*}{$o$-xylene } & 400 & 100 & 62.1 & 0.775 & 0.785 & 0.0029 & 0.0020 \\
\hline & 450 & 310 & 223 & 0.726 & 0.736 & 0.0091 & 0.0068 \\
\hline & 500 & 740 & 593 & 0.670 & 0.680 & 0.022 & 0.018 \\
\hline & 550 & 1590 & 1320 & 0.604 & 0.613 & 0.0505 & 0.041 \\
\hline & 595 & 2840 & 2431 & 0.513 & 0.522 & 0.0845 & 0.087 \\
\hline \multirow[t]{5}{*}{$m$-xylene } & 398 & 90 & 68.0 & 0.775 & 0.771 & 0.0031 & 0.0024 \\
\hline & 451 & 340 & 259 & 0.723 & 0.720 & 0.0100 & 0.0082 \\
\hline & 503 & 900 & 707 & 0.665 & 0.658 & 0.0270 & 0.022 \\
\hline & $5+7$ & 1600 & 1414 & 0.607 & 0.592 & 0.0547 & 0.046 \\
\hline & 582 & 2530 & 2285 & 0.540 & 0.515 & 0.0926 & 0.0835 \\
\hline \multirow[t]{5}{*}{$p$-xylene } & 397 & 104 & 67.6 & 0.762 & 0.767 & 0.0041 & 0.0025 \\
\hline & 452 & 370 & 268 & 0.705 & 0.711 & 0.0120 & 0.0082 \\
\hline & 499 & 800 & 664 & 0.651 & $\mathrm{X} / \mathrm{A}$ & 0.0250 & 0.020 \\
\hline & 553 & 1750 & 1544 & 0.566 & $\mathrm{~N} / \mathrm{A}$ & 0.0517 & 0.0507 \\
\hline & 584 & 2600 & 2346 & 0.504 & $\mathrm{~N} / \mathrm{A}$ & 0.1016 & 0.0875 \\
\hline \multirow[t]{4}{*}{ napthalene } & 550 & 440 & $\mathrm{~N} / \mathrm{A}$ & 0.769 & 0.771 & 0.0138 & $N / A$ \\
\hline & 600 & 920 & $\mathrm{~N} / \mathrm{A}$ & 0.721 & 0.718 & 0.0284 & $\mathrm{~N} / \mathrm{A}$ \\
\hline & 650 & 1590 & $\mathrm{~N} / \mathrm{A}$ & 0.655 & 0.655 & 0.0495 & $\mathrm{~N} / \mathrm{A}$ \\
\hline & 700 & 2600 & $\mathrm{~N} / \mathrm{A}$ & 0.570 & 0.572 & 0.0852 & $\mathrm{~N} / \mathrm{A}$ \\
\hline
\end{tabular}


Table S3: Compositions for the Binary Mixtures of Ethene/n-Heptane at $T=$ $433 \mathrm{~K}$ and Benzene $/ n$-Pentane at $T=318.15 \mathrm{~K}$ Calculated for the TraPPE-UA Force Field

\begin{tabular}{lccc}
\hline System & $p[\mathrm{MPa}]$ & $x_{\text {liq }}$ & $x_{\text {vap }}$ \\
\hline ethene $/ n$-heptane & 1.379 & 0.064 & 0.534 \\
& 2.758 & 0.166 & 0.732 \\
& 6.895 & 0.441 & 0.831 \\
& 8.274 & 0.509 & 0.828 \\
\hline$n$-pentane/benzene & 0.49 & 0 & 0 \\
& 0.898 & 0.161 & 0.448 \\
& 1.265 & 0.281 & 0.621 \\
& 1.564 & 0.469 & 0.770 \\
& 1.763 & 0.670 & 0.869 \\
2.04 & 1 & 1 \\
\hline
\end{tabular}



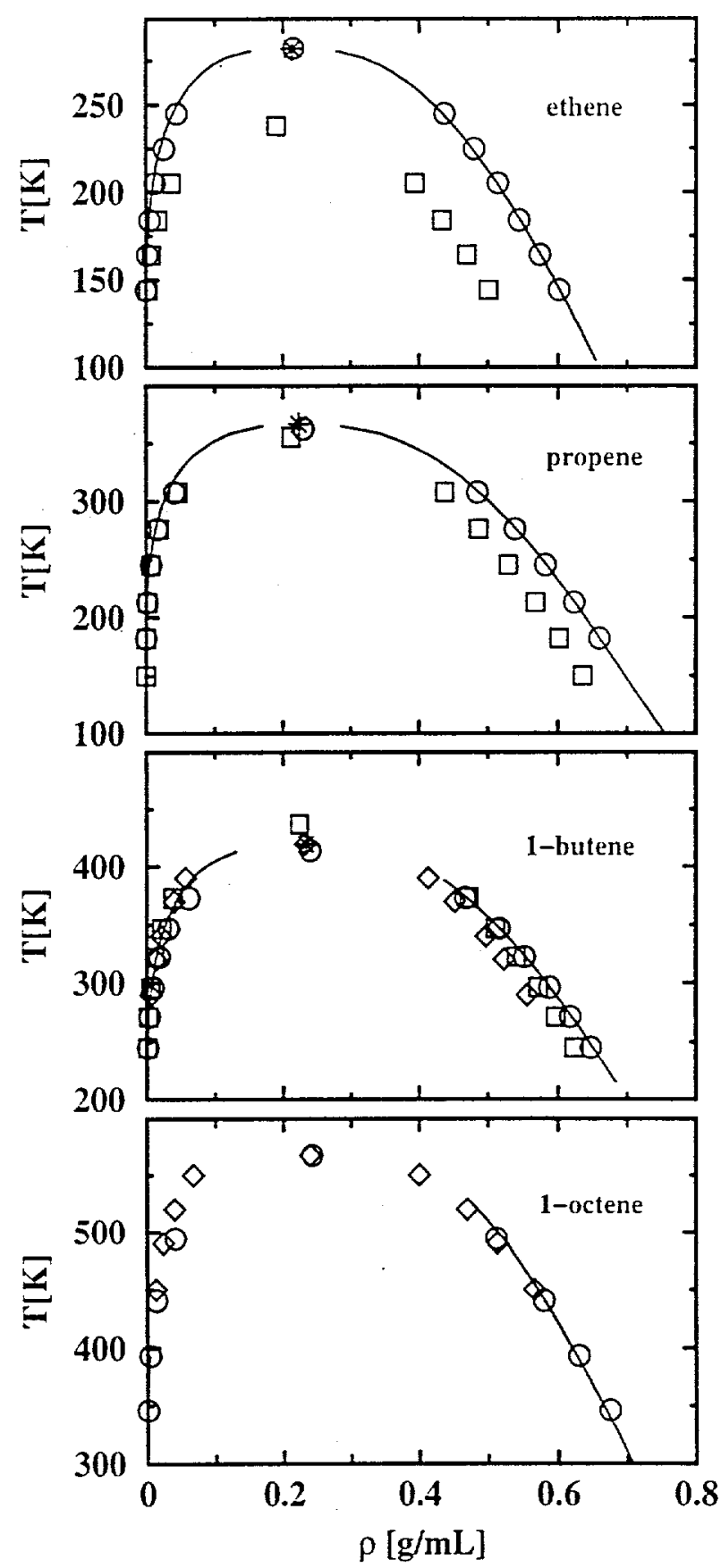

1. ij. 1 


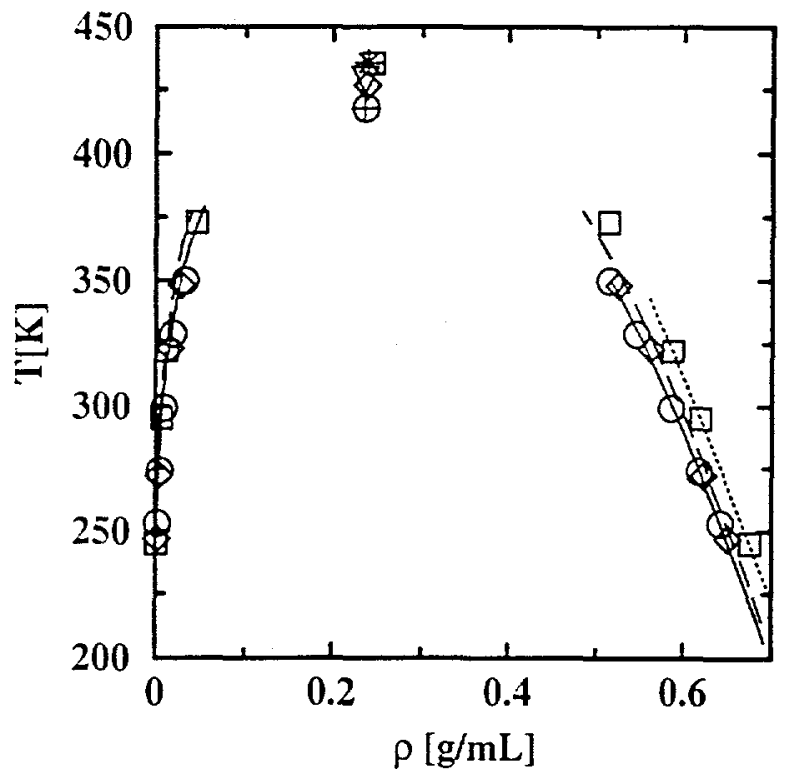

Fig. 2 


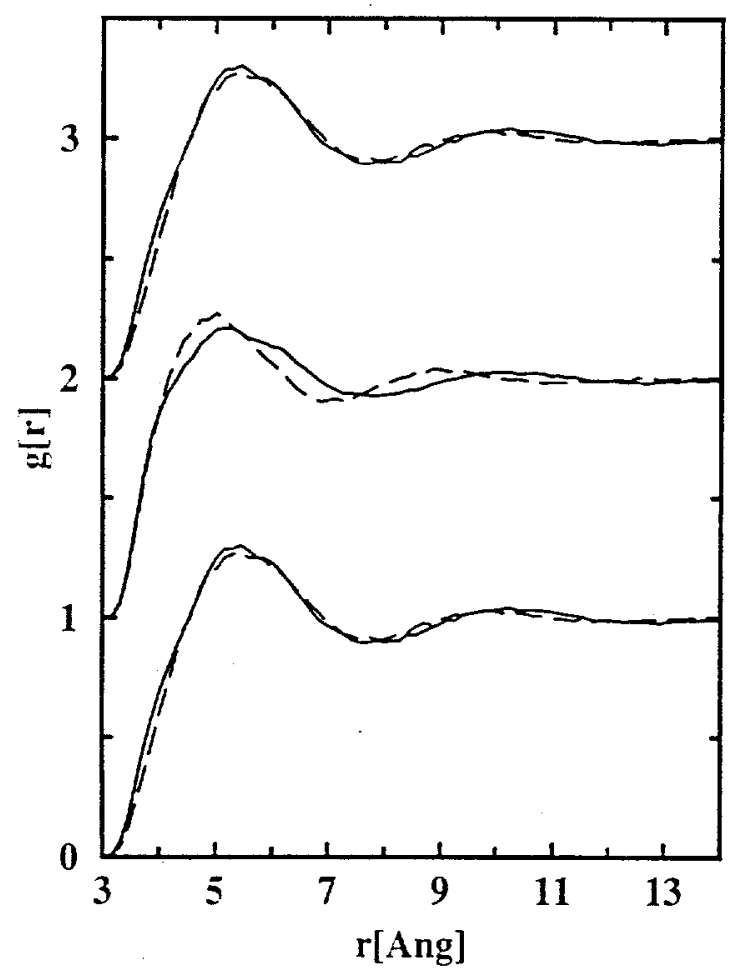

Fig. 3 


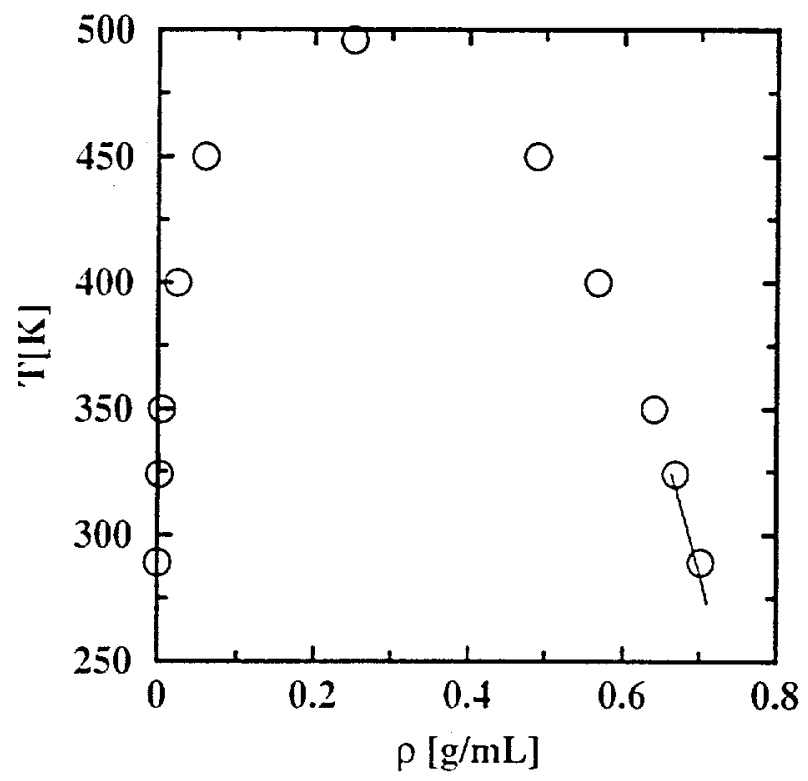

Fig. 4 

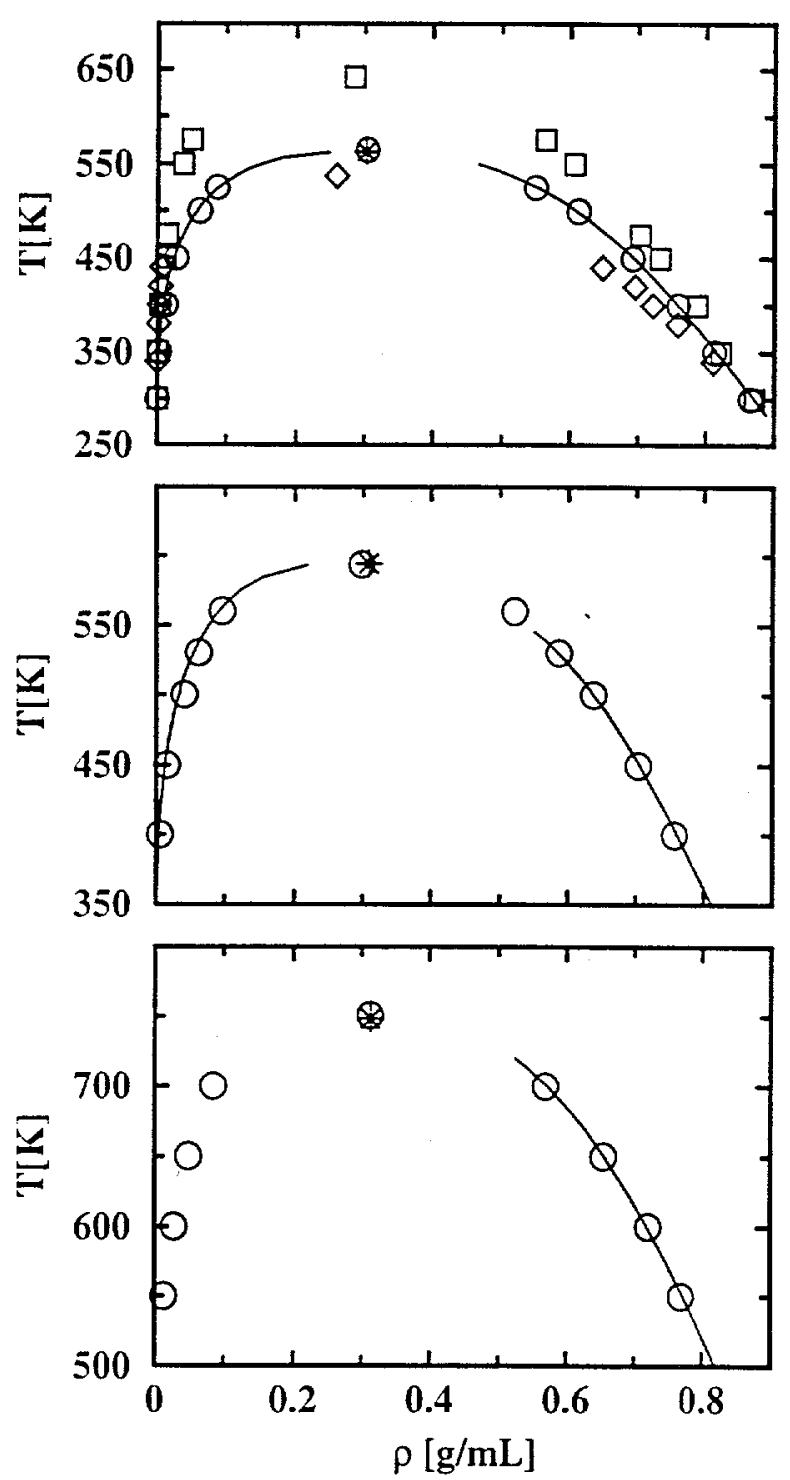

Fig. 5 


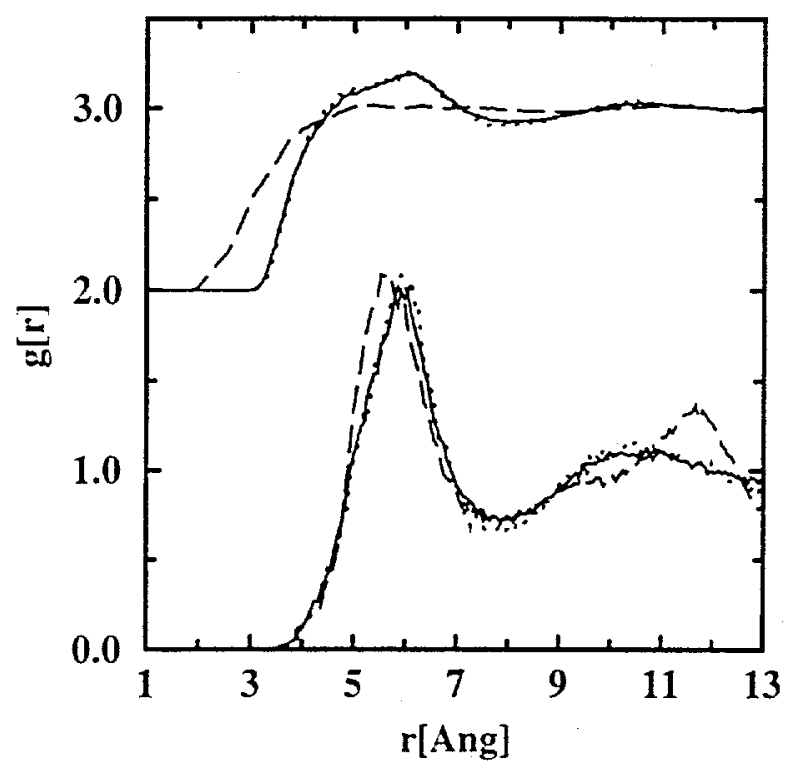

$F i g . E$ 


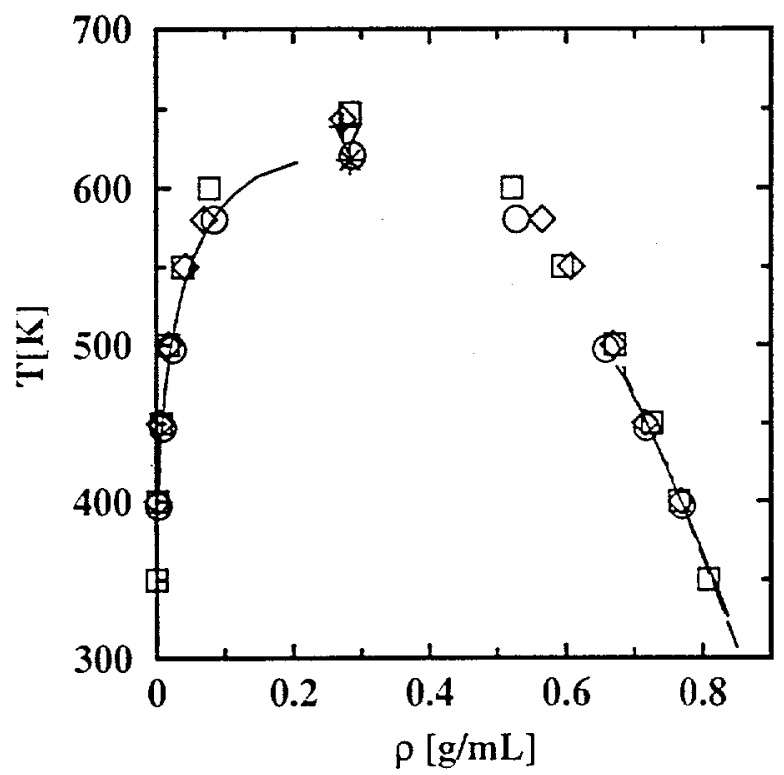

fig. 7 


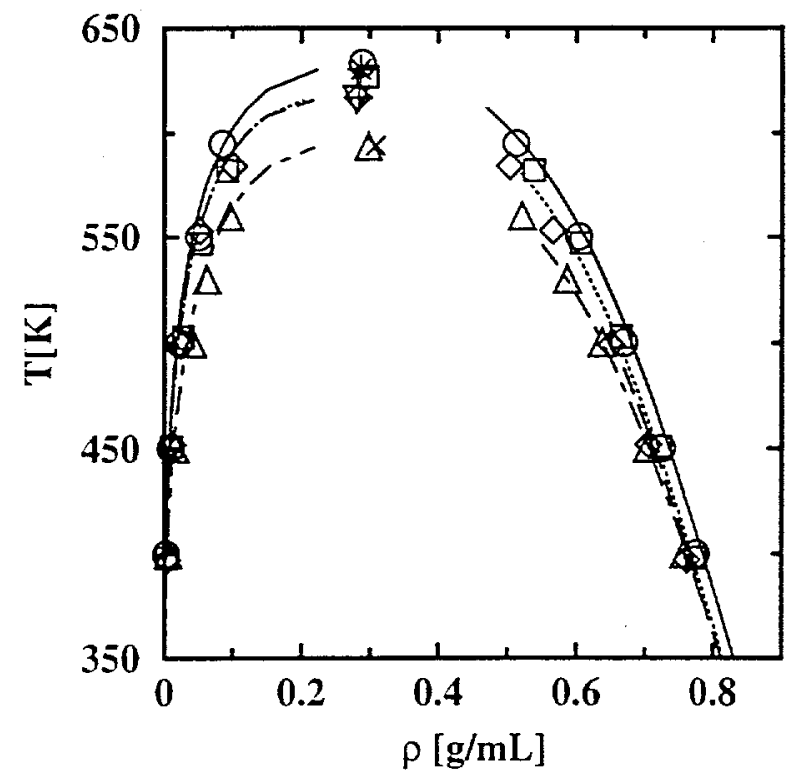

$f i g .8$ 


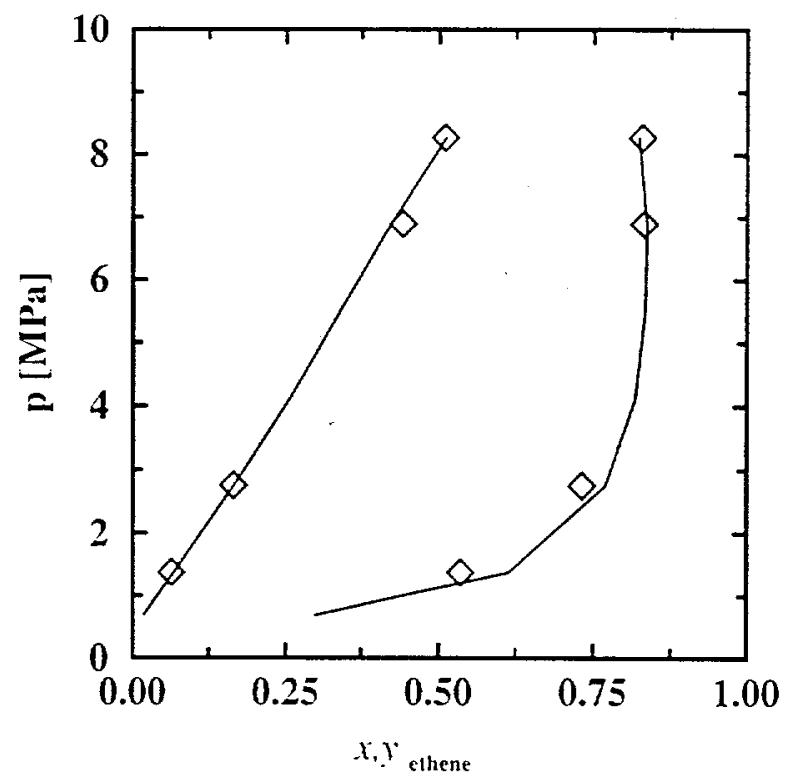

Fig. 9 



Fig. 10 

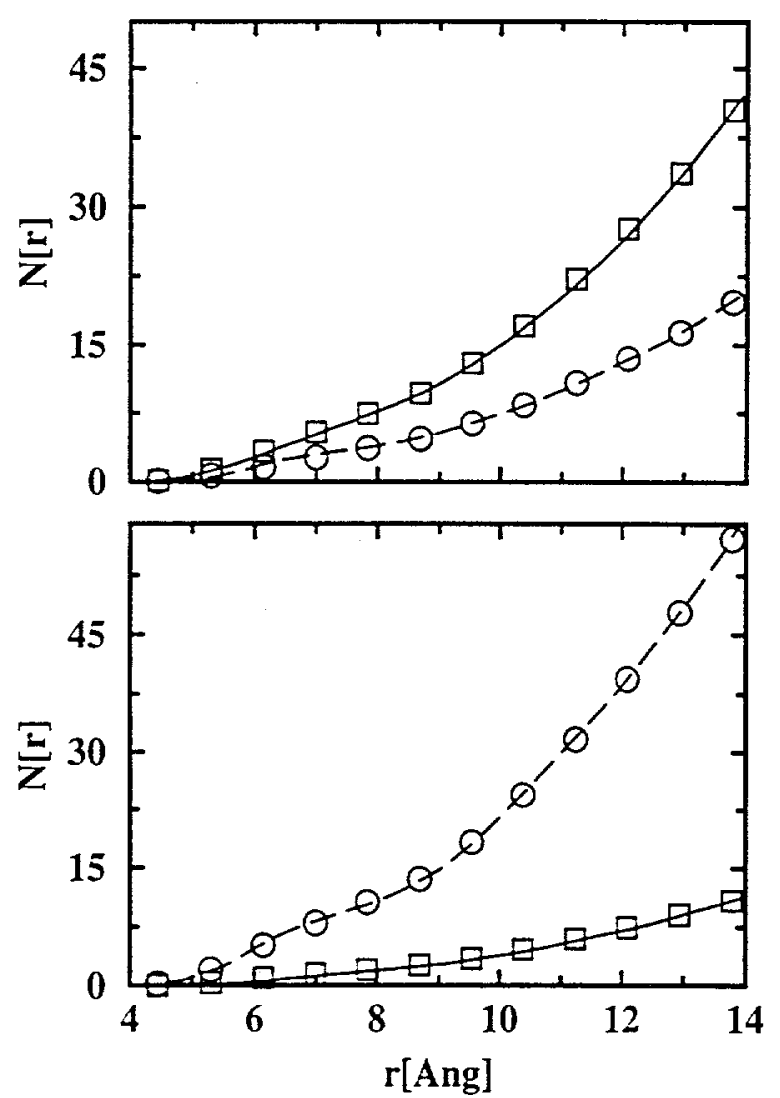

Fig. II 